\title{
Effectiveness of current physiotherapy in carpal tunnel syndrome
}

\begin{abstract}
Carpal Tunnel Syndrome (CTS) is a constellation of symptoms associated with compression of the median nerve at the wrist in the carpal tunnel. The main symptoms of CTS are numbness, pain and tingling of the first three fingers and radial side of the ring finger, nocturnal awakening is due to pain and impaired fine motor control because of weakness of the hand. Until now, the study results in which conservative methods have been applied in the treatment of CTS are contradictory. CTS rehabilitation includes laser therapy, ultrasound therapy, manual therapy, neurodynamic techniques, functional massage, splint, exercises etc. Alternative treatments for CTS include: acupuncture, massages, the Chinese cupping massage. The aim of this review is to explain the current physiotherapy methods applied in CTS patients and the research results on this subject.
\end{abstract}

Keywords: carpal tunnel syndrome, physical therapy, rehabilitation, manual therapy, splint
Volume 5 Issue 3 - 202I

\author{
Ferhan Soyuer \\ Physiotherapy and Rehabilitation Department, Faculty of Health \\ Sciences, Antalya Bilim University, Turkey
}

Correspondence: Ferhan Soyuer, Physiotherapy and Rehabilitation Department, Faculty of Health Sciences, Antalya Bilim University,Turkey, Email soyuerferhan@gmail.com

Received: April 23, 202I | Published: May 31, 2021

\section{Introduction}

Carpal Tunnel Syndrome (CTS) is the result of an compression, irritation or stretching of the median nerve as it passes through the carpal tunnel in the wrist. Its incidence has been reported to be between 0.125 and $1 \%$. CTS most often occurs between 40-60 years of age and affects ten times more often women than men. Its prevalence is between $3 \%$ and $3.4 \%$ in females and between $0.6 \%$ and $2.7 \%$ in males. ${ }^{1,2}$ In etiology; There are secondary causes such as endocrine system diseases, infiltrative lesions in the carpal tunnel, tumoral formations, traumatic events, connective tissue and rheumatic diseases and pregnancy. Although there are many diseases that cause CTS, about half of the cases are Idiopathic., ${ }^{2,3}$ Symptoms; The most important feature is paresthesia and pain at night. Pain and paresthesia that awaken the patient from sleep are not limited to the hand and can spread to the forearm and shoulder. The patient usually relieves this pain by shaking his hand. Delayed treatment in CTS may worsen the symptoms and progresses to permanent sensory loss and thenar paralysis. In moderate to severe CTS there will be hand clumsiness, weakness of thumb and atrophy of thenar muscles may be seen which will eventually decrease the functional ability of the hand and interfere with daily activities. ${ }^{2,3}$

Physiotherapeutic approaches in the carpal tunnel syndrome focus on decompression of the median nerve within the carpal canal. Until the last 5-10 years, CTS physiotherapy was planned as conservative treatments. Research results on this treatment efficacy are not clear. However, in recent years, different methods have begun to be used in CTS physiotherapy and research results are also entering the literature.

\section{Aims of physiotherapy and rehabilitation in CTS}

It is the reduction of the median nerve compression in the carpal tunnel in carpal tunnel syndrome, to ensure the continuity of ROM, reduce pain and paresthesias, maintaining and increasing muscle strength, maintaining hand functions and patient education. Considerations when determining the treatment method; Severity of symptoms, duration of symptoms, previous treatment, age of the patient, opportunity for activity changes and job modifications, cooperation of the patient. ${ }^{3,4}$ CTS rehabilitation includes laser therapy, ultrasound, splint, exercises, manual therapy, neurodynamic techniques, functional massage etc. Alternative treatments for carpal tunnel syndrome include: acupuncture, massages, the Chinese cupping massage..$^{2-4}$

\section{Physiotherapy in carpal tunel syndrome}

Treatment of CTS should be started as early as possible. An optimal combination of physical factors and kinesiotherapy is sought, depending on the disease stage, the severity of the symptoms, the proven objective changes, the individual characteristics of the patient, and accompanying diseases. Typically, a 10-day course of physiotherapy is carried out, which can be repeated several times after a break of 2-4 weeks. The functionality of the affected hand can be restored by appropriate physiotherapy rehabilitation programs. ${ }^{4-6}$

1. Splint Application; It is recommended to use wrist splints in neutral position at night in addition to conservative treatments, in patients with severe night time complaints. The purpose of splinting the wrist in neutral position is to increase the carpal tunnel volume and to reduce the pressure on the median nerve. ${ }^{7,8}$ The most preferred method in the treatment of CTS is volarassisted neutral splints and it is typically applied in the first stage. However, there is controversy in the literature about the efficacy of splint application in the treatment of CTS. It is reported that the splinting of the wrist at the neutral level reduced symptoms in $67 \%{ }^{7,8}$

Edema Control; Cold application, massage, elevation, bandage and compression. Electrotherapy agents; High voltage galvanic current, interferential current $(0-100 \mathrm{~Hz})$, russian current, fluidotherapy, traction, prolonged stretching.

Pain Control; Cold application. Electrotherapy agents; TENS, ultrasound, Phonophoresis, Interferential Current (sbt 100), Laser, shortwave diathermy, microwave diathermy, a low-frequency impulse magnetic field is assigned with parameters of 20-25 mT with period/ break ratio 2/8. Magnetotherapy, Shock-wave Therapy (SWT) is considered to be one of the non-invasive and evidence-based physical approaches to the treatment of CTS. It uses pneumatically generated shock waves with low frequency $(5-20 \mathrm{~Hz})$ and pressure of 1-5 bar applied locally in the affected area. SWT is applied in the area of 
ligamentum carpi transversalis. The therapeutic course consists of 4-6 procedures, with 1-2 procedures per week. It is particularly effective in the early stages of the disease and in young patients where the CTS is associated with occupational overload, Whirlpool, etc.

Exercise; Active-assistive, active, resistant exercises and PNF techniques are used. Stretching exercises specific to CTS are as follows; Median Nerve Stretching (provides mobilization of the $n$. medianus), Tendon Gliding Exercises (It is performed in order to prevent the adhesion and compression of the $n$. medianus in the carpal tunnel during immobilization).

Nerve Gliding Exercises (It is done to prevent the adhesion of the n. medianus in the carpal tunnel during immobilization. N. medianusa provides mobility). ${ }^{9-11}$

However, there is no evidence about the frequency, duration and intensity of exercises.

Traction; Traction application has 3 purposes in Physiotherapy.

I. Immobilization

II. Resolving muscle spasm

III. Separating joint faces with sufficient pulling force.

Desensitization and Sensory Education; Desensitization applied for hypersensitive areas is started before sensory training. The aim of desensitization is to gradually increase the patient's tolerance to touch in hypersensitive areas against stimulation. Another aim is to help the patient to reach the highest level of function with increased tolerance to touch in the hypersensitive area.

Kinesio taping (KT) banding; It is an elastic bandage that creates micro-folds in the skin, raises the skin tissue, increases blood flow, reduces pain in muscle- joint injuries and relieves compression. Application direction, duration, frequency and degree of tension are important for kinesiotaping. Improvement in pain severity, symptom severity, daily hand functions and activities of daily living was found in CTS patients with Kinesiotape taping technique.

The application of Kinesio taping in CTS significantly reduces the pressure on the nerve by lengthening the transverse carpal ligament. It reduces muscle spasms, facilitates the movement of tendons and fascia, and reduces pain through neurological suppression. Kinesio taping does not interfere with the daily work of the patient when compared to the splint, and since it has no side effects and patient compliance is high, it may be preferred in mild to moderate CTS and in patients who cannot use longterm splints. Patients are taught how to perform kinesiotherapy including self-massage at home for a short duration, frequently and with low intensity. ${ }^{12,13}$

Daily Life Activity Education; Patients with CTS should avoid excessive flexion and extension movements of their wrists in their daily lives. Modifications are essential to prevent them and to become independent in the daily life of the patient. Suit up, Hygiene, Nutrition.

Professional Rehabilitation; Job analysis should be done by examining the working environment of the patient, and the tools used at work should be modified.

Patient Education; Avoiding wrist flexion and extension, avoiding hard clutches, not holding or carrying objects for a long time, avoid catching with fingers, avoiding repetitive hand and wrist movements such as knitting or sewing, driving, avoiding activities and hobbies such as golf, tennis, table tennis, supporting the wrists during activity, do their work while resting at the computer, ensuring proper posture and conformity. ${ }^{13,14}$
The initial phases of the conservative treatment methods require corrective splints in the wrist while neutral or in an extension position, electrotherapy with ultrasound or laser, or manual therapy and exercises. At severe stages, it was recommended surgical procedures. Acupuncture has been found to be more effective in treating patients suffering from mild or moderate carpal tunnel syndrome, compared to anti-inflammatory therapy, in terms of both symptom improvement and treatment safety. ${ }^{15-17}$ Again, facial manipulations have been shown to result in better recovery in patients with carpal tunnel syndrome compared to the results obtained with low-level laser therapy. ${ }^{12}$

\section{Conclusion}

CTS is a condition that requires physiotherapy and rehabilitation. Although CTS is common and causes significant disability, it is understood from the literature that there is no consensus on its treatment. Therefore, it seems most appropriate to create individual physiotherapy and rehabilitation programs for each patient, taking into account factors such as clinical picture, accompanying diseases, age and professional background.

\section{Acknowledgments}

None.

\section{Conflicts of interest}

None.

\section{References}

1. Burton CL, Chesterton LS, Chen Y, et al. Clinical course and prognostic factors in conservatively managed carpal tunnel syndrome: A Systematic Review. Arch Phys Med Rehabil. 2016;97(5):836-852.

2. American Academy of Orthopaedic Surgeons. Management of Carpal Tunnel Syndrome Evidence-Based Clinical Practice Guideline. Accessed December 20, 2018. p. 1-95.

3. Thiese MS, Gerr F, Hegmann KT et al. Effects of varying case definition on carpal tunnel syndrome prevalence estimates in a pooled cohort. Arch Phys Med Rehabil. 2014;95(12):2320-2326.

4. Alfonso C, Jann S, Massa R, Torreggiani A. Diagnosis, treatment and follow-up of the carpal tunnel syndrome: a review. Neurol Sci. 2010;31(3):243-252.

5. Maddali Bongi S, Signorini M, Bassetti M, et al. A manual therapy intervention improves symptoms in patients with carpal tunnel syndrome: a pilot study. Rheumatol Int. 2013;33(5):1233-1241.

6. Martins RS, Siqueira MG. Conservative therapeutic management of carpal tunnel syndrome. Arq Neuropsiquiatr. 2017;75:819-824.

7. Gerritsen AA, de Vet HC, Scholten RJ, et al. Splinting vs surgery in the treatment of carpal tunnel syndrome: a randomized controlled trial. JAMA. 2002;288(10):1245-1251.

8. Page MJ, Massy-Westropp N, O'Connor D. Splinting for carpal tunnel syndrome. Cochrane Database Syst Rev. 2012;(7):CD010003.

9. Horng YS, Hsieh SF, Tu YK, et al. The comparative effectiveness of tendon and nerve gliding exercises in patients with carpal tunnel syndrome: a randomized trial. Am J Phys Med Rehabil. 2011;90(6):435-442.

10. Awan WA, Babur MN, Ansari M, et al. Effectiveness of stretching of the flexor retinaculum with ultrasonic therapy in the management of carpal tunnel syndrome. Pakistan Journal of Neurological Sciences. 2014;9(4):5-8.

11. Wolny T, Saulicz E, Linek P, et al. Effect of manual therapy and neurodynamic techniques vs ultrasound and laser on 2PD in patients with CTS: A randomized controlled trial. J Hand Ther. 2016;29(3):235-245. 
12. Pratelli E, Pintucci M, Cultrera P, et al. Conservative treatment of carpal tunnel syndrome: Comparison between laser therapy and fascial manipulation. Journal of Bodywork Movement Ther . 2015;19(1):113-118.

13. Fernandes-de-las Penas C, Ortega- Santiago R, De la Llave- Rincon AI, et al. Manual Physical Therapy Versus Surgery for Carpal Tunnel Syndrome: a Randomized Parallel-Group Trial. The Journal of Pain. 2015;16 (11):1087-1094.

14. Lim YH, Chee DY, Girdler S, et al. Median nerve mobilization techniques in the treatment of carpal tunnel syndrome: A systematic review. Journal of Hand Ther. 2017;30(4):397-406.
15. Fernández-de-las Peñas C, Ortega-Santiago R, de La Llave-Rincón AI Manual physical therapy versus surgery for carpal tunnel syndrome: a randomized parallel-group trial. J Pain. 2015;16(11):1087-1094.

16. Hadianfard H, Bazrafshan E, Momeninejad H, et al. Efficacies of acupuncture and antiinflammatory treatment for carpal tunnel syndrome. Journal of Acupuncture and Meridian Studies. 2015; 8(5):229-235.

17. Yang CP, Hsieh CL, Wang NH, et al. Acupuncture in patients with carpal tunnel syndrome: a randomized controlled trial. Clin J Pain. 2009;25:327333. 